\title{
Fagfellevurderere 2014
}

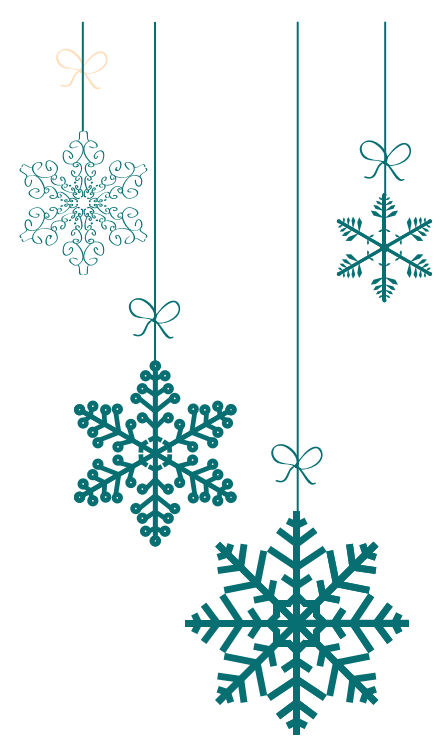

Abildsnes, Eirik Alfsen, G. Cecilie Angelsen, Anders Asfeldt, Anne Mette Aurlien, Dag

Bachmann, Ingeborg

Backe, Bjørn

Bahus, Marianne

Bartnes, Kristian

Becker, Frank

Bendiksen, Cecilie

Benestad, Haakon

Berentsen, Sigbjørn

Bergan, Stein

Bergh, Sverre

Berild, Dag

Berntsen, Erik Magnus

Berntsen, Gro

Berstad, Audun

Bindoff, Laurence

Bjerve, Kristian

Bjørge, Line

Bjørk, Marte-Helene

Bjørnelv, Sigrid

Bjørner, Trine

Bondevik, Hilde

Braarud, Anne Cathrine

Bramness, Jørgen

Brandstorp, Helen
Brantsæter, Arne

Bratland, Svein

Brekke, Mette

Bretthauer, Michael

Broch, Kaspar

Brommeland, Tor

Brustugun, Odd Terje

Bruun, Johan

Bruusgaard, Dag

$B \varnothing$, Siri Hylleraas

Carlsen, Siri

Celius, Elisabeth

Christensen, Monika

Claussen, Bjørgulf

Dahl, Alv

Dale, Ola

Diaz, Esperanza

Due-Tønnessen, Paulina

Duvaland, Lars

Ebbing, Cathrine

Eberhard-Gran, Malin

Eggen, Anne Elise

Eggesbø, Heidi

Eikeland, Randi

Ekeberg, Øivind

Ellingsen, Christian

Elverland, Hans

Engedal, Knut

Engelsen, Bernt

Eskild, Anne

Espeland, Ansgar

Finset, Arnstein

Flaatten, Hans

Flottorp, Signe

Fors, Egil A.
Fretheim, Atle

Frich, Jan

Frich, Lars

Friis, Svein

Fure, Brynjar

Gilhus, Nils

Gjelstad, Svein

Gjesdal, Sturla

Gramstad, Lars

Grewal, Harleen

Grimsgaard, Christian

Grimsrud, Tom Kristian

Gude, Tore

Gudim, Hilde Beate

Gulbrandsen, Pål

Gulseth, Hanne

Guttormsen, Anne

Haarr, Dagfinn

Haave, Per

Hafting, Marit

Hagen, Gaute

Hager, Anders

Hagestad, Kristian

Hagve, Tor-Arne

Hald, John

Hammerstrøm, Jens

Hannestad, Yngvild

Hanoa, Rolf

Harstad, Ingunn

Hasle, Gunnar

Haug, Bjørn

Haug, Jon

Haugaa, Kristina

Haugen, Arvid

Haukås, Einar

Heier, Hans

Helgeland, Jon
Helland, Åslaug

Hellebostad, Marit

Helvik, Anne-Sofie

Hervig, Tor

Hjartåker, Anette

Hjellvik, Vidar

Hjelmesæth, Jøran

Hjetland, Reidar

Hoff, Jana

Hoff-Olsen, Per

Hofvind, Solveig

Hokland, Bjørn

Holmaas, Gunhild

Hotvedt, Ragnar

Houge, Gunnar

Hrubos-Strom, Harald

Husebekk, Anne

Hyldmo, Per Kristian

Høiseth, Gudrun

Høye, Anne

Ingebrigtsen, Tor

Irtun, Øivind

Jahnsen, Jørgen

Jenum, Anne

Johansen, May-Lill

Johansen, Rune

Jørgensen, Anders Palmstrøm

Kalager, Mette

Karstensen, Anne

Kirkevold, Øyvind

Kiserud, Torvid

Kjekshus, Lars Erik

Kjellmo, Åse

Kringlen, Einar

Kristiansen, Ivar Sønbø

Krohn, Jørgen 


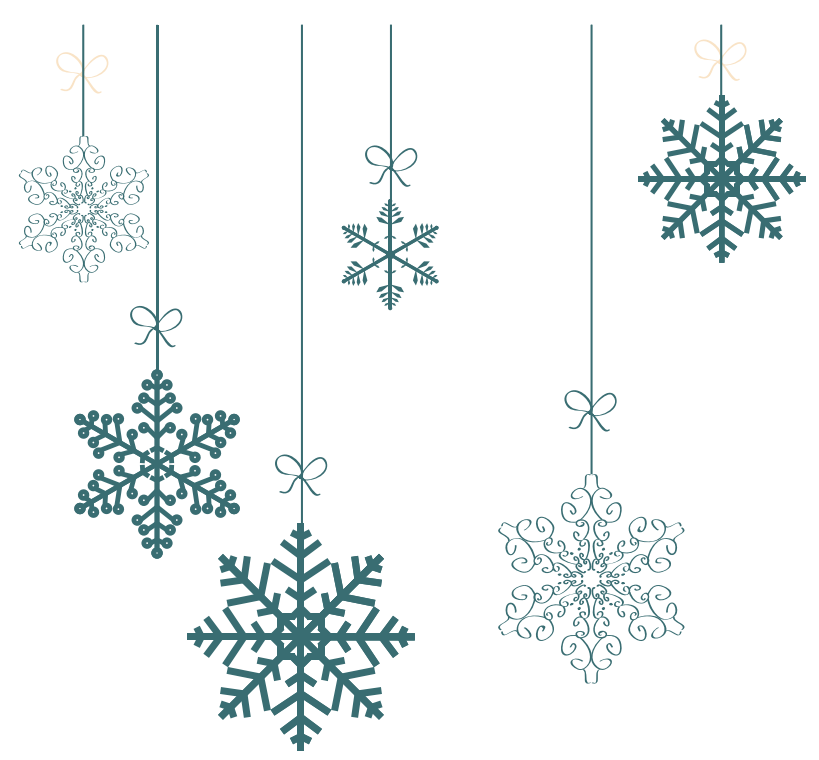

Kulseng, Bård

Kurz, Kathinka

Kvernmo, Hebe

Kvistad, Kjell

Kørner, Hartwig

Lagerløv, Per

Lang, Astri Maria

Larsen, Alf Inge

Lassen, Kristoffer

Lekven, Jon

Lien, Lars

Lillestøl, Kristine

Lindenskov, Paal

Lund, Karl Erik

Lund, May Brit

Magnussen, Jon

Mala, Tom

Mehlum, Lars

Meland, Eivind

Midtvedt, Øyvind

Moen, Mette

Molven, Anders

Munk, Peter Scott

Myrvang, Bjørn

Møller, Pål

Mørland, Jørg

Nakken, Karl Otto

Nakstad, Per

Natås, Olav

Nedrebø, Bjørn Steinar Olden

Nes, Harald

Ness, Ewa

Nestvold, Torunn

Nielsen, Erik

Nord, Erik

Nordbø, Svein
Norderhaug, Inger

Nordfalk, Karl Fredrik

Nordrehaug, Jan Erik

Norheim, Ole

Nygaard, Øystein

Nylander, Gro

Nylenna, Magne

Næss, Halvor

Opdal, Mimi

Paus, Benedicte

Pihlstrøm, Lasse

Pripp, Are

Qureshi, Samera Azeem Qvigstad, Elisabeth

Randsborg, Per-Henrik

Ranhoff, Anette

Rao, Vidar

Ree, Anne Hansen

Reed, Wenche

Reiso, Harald

Rise, Marit

Risøe, Cecilie

Robsahm, Trude

Rognum, Torleiv Ole

Rosland, Jan Henrik

Ruud, Ellen

Ræder, Johan

Rørtveit, Sverre

Saltvedt, Ingvild

Salvesen, Rolf

Sand, Trond

Sandbæk, Gunnar

Sando, Sigrid Botne

Sandvei, Per
Sandvik, Leiv

Schirmer, Henrik

Selmer, Randi

Skeie, Ivar

Skeie, Geir Olve

Skjeldestad, Finn

Skrede, Steinar

Skretting, Astrid

Slåstad, Siri

Solberg, Steinar

Sommervoll, Dag Einar

Spigset, Olav

Staff, Annetine

Steinsvåg, Sverre

Storstein, Anette

Straand, Jørund

Strømmen, Magnus

Sundsfjord, Arnfinn

Sundstrøm, Stein $H$.

Swensen, Elisabeth

Søreide, Eldar

Søreide, Jon Arne

Søreide, Kjetil

Tangen, Jon

Thelle, Dag Steinar

Thomassen, Lars

Thoresen, Magne

Tjora, Solve

Tjønnfjord, Geir

Tysnes, Ole-Bjørn

Tønnessen, Tor Inge

Uhlig, Till

Vaglum, Per

Vandvik, Inger Helene

Vandvik, Per Olav

Vedeler, Christian
Viste, Kristin

Vollset, Stein Emil

Waage, Anders

Waal, Helge

Walberg, Mette

Wensaas, Anders

Wensaas, Knut-Arne

Wergeland, Ebba L.

Wester, Knut Gustav

Wibe, Torunn

Wiseth, Rune

Wyller, Torgeir

Ødegård, Asbjørn

Øiesvold, Terje

Ørstavik, Kristin

Aaberge, Ingeborg

Aamodt, Geir

Aaraas, Ivar Johannes

Aarli, Ånen

Aasebø, Ulf

Aasen, Tor Olav

Aasheim, Erlend

Aasland, Olaf

Aasly, Jan

Aavitsland, Preben 